\title{
La inteligencia emocional en niños y jóvenes con necesidades educativas especiales de tipo intelectual
}

\author{
Emotional intelligence in children and young people with special \\ intellectual educational needs
}

\begin{abstract}
Resumen
Esta investigación se basa en una revisión sistemática sobre la inteligencia emocional y las personas con necesidades especiales de tipo intelectual. Realizándose una descripción sobre los diferentes modelos teóricos e implicación educativa actual, con el objetivo de describir las investigaciones que justifican la necesidad de su desarrollo en personas con discapacidad intelectual. Utilizando las bases de datos Google Académico y Dialnet se han obtenido 25 artículos, los cuales describen sus beneficios para el bienestar personal, la autoeficacia, la autoestima, la confianza y la seguridad que les proporciona. El desarrollo de la inteligencia emocional les proporciona mayor satisfacción vital y calidad de vida debido a que facilita un mayor conocimiento, control y regulación emocional, convirtiéndose estos en recursos para afrontar los impulsos, la ansiedad, la frustración y el estrés de estas personas. Por tanto, se convierte en un eslabón más en su desarrollo y formación integral, facilitándoles y mejorando su desarrollo y relaciones interpersonales.
\end{abstract}

\section{Palabras clave}

Inteligencia emocional, discapacidad intelectual, emociones, desarrollo integral.

\begin{abstract}
This research is based on a systematic review on emotional intelligence and people with special intellectual needs. Making a description about emotional intelligence, theoretical models and current educational involvement, with the aim of describing the research that justifies the need for the development of emotional intelligence in people with intellectual disabilities. Using the Google Academic and Dialnet databases, 25 articles were obtained, which describe the benefits of emotional intelligence for personal well-being, self-efficacy, self-esteem, confidence and security that it provides. The development of emotional intelligence provides them with greater life satisfaction and quality of life because it facilitates greater knowledge, control and emotional regulation, turning these into resources to face the impulses, anxiety, frustration and stress of these people. Emotional intelligence is one more link in their development and comprehensive training, facilitating and improving their development and interpersonal relationships.
\end{abstract}

\section{Keywords}

Emotional intelligence, intellectual disability, emotions, integral development.

\author{
Daniel Garrote Rojas \\ <danielgarrote@ugr.es> \\ Universidad de Granada. España

\section{Carla Castro Garrote} \\ $<$ carlacastro95@gmail.com>
}

\section{Sara Jiménez-Fernández} <sarajimenezfer@hotmail.com>

Hospital Universitario de Jaén. España

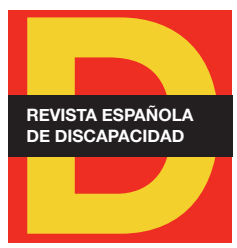

Para citar:

Garrote, D. et al. (2021). La

inteligencia emocional en niños y jóvenes con necesidades educativas especiales de tipo intelectual. Revista Española de Discapacidad, 9(2), pp. 119-134.

Doi: <https://doi.org/10.5569/23405104.09.02.07>

Fecha de recepción: 27-10-2020 Fecha de aceptación: 19-09-2021 


\section{Introducción}

La tendencia e influencia de diversos autores y sus modelos de evaluación de la inteligencia emocional se han convertido en uno de los fenómenos que paulatinamente va tomando mayor presencia y cabida en diferentes entornos y contextos de la sociedad del siglo XXI, entre ellos, el educativo (Fernández-Berrocal y Extremera, 2002). Las instituciones educativas, el currículum de educación y otras entidades son los encargados de implementar diversos contenidos y acciones metodológicas, entre otros aspectos, que tienen la obligación de dar respuesta a las demandas inconscientemente requeridas y propuestas por la sociedad que la componen; es decir, como los autores Mella y Ortiz (1999) indican, la sociología determinista de la educación (...) lleva a la negación sociológica de la escuela como una institución independiente de los efectos de la sociedad global (p. 71).

Considerando la actual ley educativa LOMLOE (Ley Orgánica de Modificación de la LOE, Ley Orgánica 3/2020, de 29 de diciembre, por la que se modifica la Ley Orgánica 2/2006, de 3 de mayo, de Educación), y la consiguiente puesta en marcha en los colegios, es difícil lograr el fomento del desarrollo integral de la persona. Además de las críticas expuestas por Trujillo y Rivas (2005), podría afirmarse que, en parte, también se debe a la escasez y presencia nula del trabajo y tratamiento de las emociones en aulas cada vez más diversas y con diferentes necesidades educativas especiales; ya que, todavía las instituciones educativas actuales se rigen por metodologías tradicionales en las que los alumnos y alumnas con mejores calificaciones son aquellos que tienen mayor cociente intelectual. Sin embargo, la inteligencia académica no es suficiente para alcanzar el éxito profesional (Fernández-Berrocal y Extremera, 2002) porque el cociente intelectual de una persona no es un indicador preciso que asegura el éxito en la vida; además, la inteligencia académica no proporciona una formación propicia para hacer frente a las dificultades (Orellana-Cortijo, 2017). La presencia del tratamiento de las emociones en la LOMCE (Ley Orgánica 8/2013, de 9 de diciembre, para la mejora de la calidad educativa) busca el desarrollo social y personal de forma transversal en el currículo.

Esta investigación busca la reivindicación de la necesidad del tratamiento y desarrollo de la inteligencia emocional en cualquier entidad educativa y aula, a consecuencia de los beneficios y aspectos positivos que tiene para ofrecer tanto individual como colectivamente a alumnos, maestros, familiares y la sociedad en general y, del mismo modo, a las personas con necesidades de tipo intelectual. Facilitando la inteligencia emocional desenvolverse mejor en el mundo (González y Villanueva, 2014), mantener relaciones saludables con adultos e iguales (Bisquerra et al., 2015), aportar una mejor adaptación personal, social, académica y laboral (Palomera et al., 2017), facilitando la detección y adaptación a los cambios con una adecuada respuesta (Bisquerra, et al., 2015). Siendo clave la regulación y control de las emociones (Jiménez y López-Zafra, 2009).

Muchos contenidos educativos tienen como base o fin el desarrollo cognitivo de sus educandos, por el motivo de que, como puede comprobarse en cualquier realidad educativa, aparecen infinidad de contenidos relacionados con capacidades memorísticas, entre otros. Además de la inteligencia, han de tenerse en cuenta otros aspectos como la afectividad y la personalidad, con la intención de poder valorar y trabajar adecuadamente la capacidad de adaptación de los individuos, manteniendo que la inteligencia tiene, del mismo modo, una estrecha relación con las emociones, así como la propia personalidad y los aspectos sociales (Chamarro y Oberst, 2004).

El ser humano está caracterizado por tres dimensiones, según las tesis de Zubiri (2006): individual, social e histórica; las cuales son exigidas por la propia realidad y necesidad como persona. Goleman (1995), por 
su parte, popularizó la idea de que la experimentación y la expresión de las emociones, como parte de la inteligencia y habilidad para la adaptación en diferentes circunstancias de la vida, es un aspecto a destacar, ya que considera que la inteligencia emocional es más determinante que el desarrollo cognitivo que una persona puede alcanzar en su vida, tanto en áreas de trabajo como en sus relaciones personales (Chamarro y Oberst, 2004). Por lo tanto, si un ser humano está compuesto por tres dimensiones, entre ellas, la social, y ésta a su vez está estrechamente relacionada con las emociones, no se debería descartar la posibilidad de poder desarrollar un aspecto tan importante que configura al ser humano. Es más, se ha comprobado, en el entorno más cercano, la necesidad de trabajar las emociones en el aula, tanto para todo el alumnado en general, como para aquellas personas con necesidades especiales; por el motivo de que el impacto de la obra de Goleman (1995) y su popularidad (Chamarro y Oberst, 2004) no ha llegado a todas las instituciones educativas de nuestra sociedad. El hecho de que no haya llegado esta divulgación e interés por el tratamiento de la inteligencia emocional a nuestros centros educativos descarta la opinión general de esa necesidad de gestión y tratamiento de las emociones que promulgan que tiene nuestra sociedad autores como Roberts, Zeidner y Matthews (2001).

El sistema educativo ha de adaptarse a la sociedad y a sus constantes transformaciones (Orellana-Cortijo, 2017), ofreciendo a todo el alumnado, tenga las habilidades y capacidades que tenga, como bien dice Acosta (2011), a través de una educación de calidad que contribuya al pleno desarrollo de su personalidad y capacidades, potenciando sus capacidades a lo largo de su etapa educativa (Álvarez et al., 2018; Fiori y Vesely-Maillefer, 2018). Del mismo modo, este autor indica que no puede concebirse una educación de calidad, donde se fomente el desarrollo integral de una persona, sin la puesta en marcha de proyectos y programas que incentiven la inteligencia emocional. El profesorado presenta una adecuada visión del concepto inteligencia emocional pero no la emplea explícitamente sino utilizando actividades basadas en un trabajo transversal a la práctica escolar (García-Tudela y Marín-Sánchez, 2021), buscando un proceso educativo que implique a toda la comunidad educativa a trabajar de forma cooperativa hacia una sociedad emocionalmente más inteligente (Pinos, 2019). El desarrollo de la inteligencia emocional en personas con necesidades especiales, en concreto en aquellas personas con necesidades de tipo intelectual, supone una respuesta directa, sobre todo, a los requerimientos sociales de estos individuos, ya que pueden presentar dificultades en el control emocional (Álvarez et al., 2018; Cabrera et al., 2016). Estas necesidades sociales concretas no están suficientemente atendidas en el currículo ordinario; aspectos como la ansiedad, la depresión, el estrés, los comportamientos de riesgo, etc. son parte de cada día en las escuelas a consecuencia del analfabetismo emocional que padecen no solamente los estudiantes con discapacidad intelectual (Caro et al., 2019). Con el transcurso de las diferentes épocas, los años e incluso las décadas, nuestra sociedad ha ido avanzando y progresando en infinidad de aspectos, entre los que cabe destacar la educación. Es cierto que, la educación no solamente se produce en las instituciones educativas, puede compartirse a través de ideas, opiniones, culturas, conocimientos, etc., y del mismo modo, puede estar presente en las propias acciones, sentimientos y actitudes (Ruiz, 2010). La evolución de la Educación Especial, por su parte, ha dependido de la manera en la que han reflejado las diferentes administraciones a las personas que requieren de este tipo de educación; donde ha pasado de atenderse a sujetos denominados como retrasados y retrasadas, desde un modelo de déficit, al modelo de atención a la diversidad o de escuela inclusiva, con carácter más abierto, interactivo y con mayores expectativas de desarrollo (González, 2009).

La educación actual ha sido conformada a consecuencia de una transición desde una escuela completamente tradicional a una escuela más actual que lucha por nociones nuevas como la diversidad y la inclusión. Todo ello se ha ido caracterizando y conformando debido a diversos cambios todavía pendientes de 
una completa consolidación; ya que nos encontramos en un proceso de recogida de información del que se esperan muchos resultados beneficiosos y crecimiento y no, precisamente, un estancamiento (Román, 2005). En cuanto a las emociones, que todavía tienen un papel muy discreto en las escuelas, Salovey y Mayer (1990) contribuyeron a que esto cambiase proponiendo el concepto de inteligencia emocional y demostrando las aportaciones beneficiosas que ésta tiene: diferentes conductas, éxito en la vida y mejor rendimiento académico, entre otras. Sus aportaciones contribuyeron a importantes avances en el desarrollo de programas de gestión emocional en los centros educativos (Buitrago y Herrera, 2013), realizándose desde las instituciones educativas numerosas experiencias y programas (Aránega et al., 2014). Esta tendencia surge a partir del siglo $\mathrm{XX}$, a consecuencia del fortalecimiento y tendencia al sentimiento y necesidad de modificación y amoldamiento a las circunstancias propias de cada tiempo, así como las notorias ganas y curiosidad del ser humano para solventar eficientemente con asiduidad las demandas del futuro. A partir del final del siglo pasado, todas estas tendencias mencionadas fueron igualmente reflejadas en el ámbito educativo debido a que surgió la necesidad de modificar los criterios pragmáticos para la intervención y actuación educativa, dando fruto a un nuevo fenómeno sociopedagógico que introdujo muchos beneficios: nuevos valores, sentimientos de pertenencia, fomento de la inclusividad, respeto hacia las diferencias y la diversidad, etc. Una de las conceptualizaciones de la educación, según Delors (1996) se fundamenta en base a cuatro pilares: aprender a hacer, a convivir, a conocer y aprender a ser. Así es que la educación tiene como fin buscar y favorecer el desarrollo de las capacidades y habilidades de cualquier persona sin excepción, intentando mitigar los aspectos que desfavorezcan esto último (concepciones sociales, prejuicios, búsqueda del éxito por encima de todo, rivalidad, entre otros).

Actualmente podría decirse que nuestra sociedad está inmersa en una crisis social, por el motivo de que sus integrantes buscan el máximo beneficio individual a nivel material, sin considerar la presencia de los demás y prevaleciendo aspectos tan degenerantes como la individualidad (Mateu, 1987). Es por esto por lo que la sociedad de hoy en día depende de una educación de calidad para poder seguir avanzando y evolucionando, fundamentándose en aspectos como el trabajo cooperativo, trabajando el currículum en todos sus niveles, incentivando la pertenencia personal y social, así como la autoestima (Braslavsky, 2004) para la obtención de un bienestar subjetivo presente y futuro de cada persona, y como este bienestar puede influir en el rendimiento de diferentes actividades (Fogarty et al., 2016), favoreciendo así la comunicación entre el profesor y los alumnos y entre los propios alumnos (Garrote et al., 2019).

La discapacidad intelectual centra su atención, según Shalock (2009), en la expresión de las limitaciones del funcionamiento individual dentro de un contexto social y representa una desventaja sustancial para el sujeto. El concepto ha evolucionado desde un rasgo de la persona (déficit) a un fenómeno humano que tiene origen en factores orgánicos y/o sociales (factores que dan origen a limitaciones funcionales que provocan cierta restricción para realizar cualquier acción que se espera en la sociedad).

Existen dos grandes grupos en los que podrían diferenciarse los modelos de la inteligencia emocional:

- Los modelos de habilidades, basados en el procesamiento de la información, corresponden a una visión funcionalista de las emociones y representan el uso que se hacen de las mismas en diferentes situaciones para que consecuentemente se apliquen en el pensamiento. El modelo más representativo es el de Salovey y Mayer (1990). Es un método que fundamenta la unión entre las emociones y el razonamiento, siendo la inteligencia emocional la que se centra en el procesamiento de la información emocional (Mayer y Salovey, 1997). Además, cabe mencionar la estrecha relación que existe con la inteligencia verbal, debido a que la expresión y comprensión de los sentimientos se ven fuertemente vinculados a ella 
(Mayer et al., 1999). Por lo tanto, es un uso adaptativo de las emociones y su aplicación al pensamiento que tenemos (Fernández-Berrocal y Extremera, 2005). Son emociones que se consideran que están en continuo cambio, por el motivo de que cada ambiente específico requiere de diferentes emociones (Sternberg y Kaufman, 1998).

- Los modelos mixtos, cuyos principales autores han sido Goleman (1995) y Bar-on (1997), están compuestos por los rasgos estables y la capacidad de automotivación de cada persona, con el objetivo de adquirir una habilidad para poder regular eficientemente las propias emociones. Están fundamentados por aspectos relacionados con la personalidad, las habilidades cognitivas y los elementos de cada persona en particular. Según este modelo, la inteligencia emocional se refiere a los comportamientos típicos y formas de experimentar que se tiene del mundo, en vez de hacer referencia a una habilidad pragmática (Trujillo y Rivas, 2005).

El modelo de habilidades, también llamado modelo de cuatro ramas de la Inteligencia Emocional (MestreNavas y Fernández-Berrocal, 2007), hace uso de una visión funcionalista de las emociones en diferentes situaciones para que después éstas sean aplicadas en el pensamiento. A través de este modelo, las emociones se consideran como aspectos en continuo cambio, porque en un ambiente específico no se requiere de las mismas emociones que en otra situación o en otra circunstancia (Sternberg y Kaufman, 1998). Este modelo de cuatro ramas se basa en cuatro ámbitos, los cuales corresponden a cuatro aspectos diferentes de las emociones. Según los investigadores Mestre-Navas y Fernández-Berrocal (2007), cada uno podría explicarse y describirse de la siguiente manera: percepción y expresión emocional, facilitación emocional, comprensión emocional y regulación emocional.

El modo de pensar de estos niños y niñas en la escuela está caracterizado por una memoria mecánica y una incapacidad de generalización. La memoria mecánica que puedan tener puede ser de alta calidad; sin embargo, no significa que entiendan lo que han recordado. Por otro lado, tienen escasa habilidad general y pensamiento, además de la dificultad para hacer distinciones entre aspectos importantes y otros no tan importantes y, habitualmente, necesitan ayuda en sus habilidades adaptativas (Gento y Strnadová, 2011). Según Rodríguez (2008) la personalidad de estas personas está bastante caracterizada. Su autoconocimiento es bastante nulo, por el motivo de que se ha afirmado que un cociente intelectual bajo conlleva un menor éxito y, por consiguiente, menor conocimiento propio y subestimación de sus capacidades. Del mismo modo, se les hace difícil reconocer e identificar sus emociones. Además, muestran mayores niveles de ansiedad que personas con la misma edad cronológica o edad mental, aunque esto último no está atestiguado como consecuencia del nivel cognitivo, sino que se debe, a priori, a factores vivenciales más que intelectuales. En cuanto a la capacidad de regular la propia conducta (capacidades de regulación, autorrefuerzo y auto-observación), habitualmente no se encuentra en estas personas, debido a que el autocontrol es una de las habilidades menos desarrolladas en ellos y ellas por la baja tolerancia a la frustración. Así mismo, las personas con necesidades especiales de tipo intelectual pueden presentar baja autoestima, a consecuencia de los posibles fracasos que han podido llegar a tener durante su vida; además de la escasa habilidad para mantener relaciones sociales, que pueden verse afectadas por comportamientos de inadaptación emocional o respuestas impulsivas o disruptivas, en ocasiones, llevándoles a situaciones de rechazo (Alonso, 2013). Así es que, la inteligencia emocional, además de beneficiar el contexto educativo, también beneficia el personal y social, desarrollando las habilidades adaptativas de estas personas y favoreciendo su desarrollo integral, para que en un futuro puedan, en la medida de lo posible, vivir independientes, sin guía ni supervisión continua, a pesar de que, en infinidad de ocasiones, las familias quizás tengan que sobreponerse a situaciones preocupantes y a la sobreprotección para el beneficio de sus descendientes. 
El contexto familiar puede sufrir con mayor frecuencia las consecuencias del estrés, provocando en ocasiones presión o tensión familiar o, incluso, alteración de su situación a causa de condiciones externas que influyen en el ecosistema familiar. Estos elementos externos provocan estrés debido a que suponen aspectos, a priori, fuera del control de las familias (Gento y Strnadová, 2011). A pesar de ello, las familias deberían favorecer la ocasión de autosuficiencia a sus hijos en edad escolar, apostando por una educación que favorezca el desarrollo integral de sus infantes, reduciendo así esta ansiedad y estrés; ya que, viendo en primera persona los beneficios que puede ofrecer la inteligencia emocional, comprobarían que sus descendientes, poco a poco, irían haciendo uso de herramientas que les ofrecerían la oportunidad de defenderse en su presente y futuro en diferentes ámbitos vitales; convirtiéndose esto último en un aspecto tranquilizador para las familias. Así es que, como puede comprobarse, la inteligencia emocional no sería únicamente beneficiosa para este colectivo objeto de estudio, sino para su entorno familiar y educativo también.

\section{Objetivo}

Como indica Paredes (2010) los aspectos emocionales y afectivos reciben muy poca atención en situaciones y ámbitos que acogen a personas con necesidades especiales de tipo intelectual, además de los escasos estudios que se han realizado con estas personas acerca de esta temática. Así es que podría indicarse que actualmente se cuenta con insuficientes documentos, investigaciones, etc. sobre la vida emocional de estos sujetos, así como estudios que relacionen este trastorno con la inteligencia emocional, ya sean, niños, adolescentes o adultos (Sánchez-Ramírez, 2018). El hecho de que en los últimos años la inteligencia emocional haya tomado paulatinamente mayor cabida y haya suscitado, así mismo, mayor interés, sobre todo en la etapa de educación primaria (Soler et al., 2018), así como en el ámbito universitario como recurso benefactor para un mejor ajuste y adaptación al nuevo medio (Suriá, 2018), demuestra el interés y efecto positivo que puede llegar a tener en ámbitos educativos.

Por lo tanto, como consecuencia de esta relativamente visible necesidad y breve consolidación en entornos educativos del siglo XXI, en esta investigación, basada en una revisión sistemática, pretendemos analizar la literatura científica para dar a conocer aquellas investigaciones que justifican la importancia y/o necesidad del desarrollo de la inteligencia emocional en jóvenes con algún tipo de necesidad especial intelectual.

\section{Metodología}

Haciendo hincapié en el objetivo y con la intención de identificar, evaluar y sintetizar los estudios más destacados que se han desarrollado acerca de la inteligencia emocional y la discapacidad intelectual se lleva a cabo una revisión sistemática a través de las bases de datos Dialnet y Google Académico. La base de datos Dialnet está considerada como uno de los portales bibliográficos más significativos de difusión científica del mundo, abordando ámbitos de conocimiento de las Ciencias humanas, jurídicas y sociales, siendo la fuente abierta de producción científica más completa en español (Mateo, 2015). 
Como criterios de selección se han utilizado las palabras clave: inteligencia emocional y discapacidad intelectual. No se han llevado a cabo restricciones de tipo temporal a consecuencia del poco tiempo que lleva investigándose la inteligencia emocional. Un límite en la búsqueda ha sido el idioma, utilizando el castellano. Aparecieron cuarenta y dos trabajos de investigación; tras un proceso de selección manual para ajustarnos a las necesidades de la revisión, descartando aquellos donde la población objeto de estudio no eran niños o jóvenes, se han analizado un total de veintisiete artículos con contenido relacionado con el área de educación emocional y personas con necesidades intelectuales entre las edades de la niñez, adolescencia y juventud.

\section{Resultados}

A través de la búsqueda descrita anteriormente, se han seleccionado 25 documentos científicos sobre la inteligencia emocional y la discapacidad intelectual como aparece en la tabla 1.

\begin{tabular}{|c|c|c|}
\hline AUTOR & MUESTRA & CONCLUSIONES \\
\hline Ruiz (2004) & $\begin{array}{l}\text { Niños, niñas } \\
\text { y jóvenes con } \\
\text { síndrome de Down }\end{array}$ & $\begin{array}{l}\text { Necesidad de introducir la educación emocional como un componente esencial } \\
\text { de la formación integral de estas personas para un conocimiento y control de sus } \\
\text { propias emociones, siendo el equilibrio emocional y la autosatisfacción personal } \\
\text { bases fundamentales para una adecuada calidad de vida. De este modo, se } \\
\text { ayudaría a que alcanzaran un grado apropiado de equilibrio personal, así como el } \\
\text { favorecimiento de su interacción social. }\end{array}$ \\
\hline $\begin{array}{l}\text { Rodríguez } \\
\text { (2008) }\end{array}$ & $\begin{array}{l}\text { Una niña de } 14 \\
\text { años y dos niños } \\
\text { de } 8 \text { y } 11 \text { años, } \\
\text { todos ellos con } \\
\text { un diagnóstico de } \\
\text { retraso mental leve }\end{array}$ & $\begin{array}{l}\text { Se observa que las habilidades de la inteligencia emocional en estas personas no } \\
\text { se evidencian con un óptimo desarrollo; en algunas se ven disminuidas y en otras, } \\
\text { incluso, no se observan. Además, clarifican que la atención oportuna, acompañada } \\
\text { de programas de desarrollo de inteligencia emocional evitaría y atenuaría } \\
\text { situaciones desfavorables para la calidad de vida de estos niños y niñas. }\end{array}$ \\
\hline Paredes (2010) & $\begin{array}{l}\text { Personas con } \\
\text { discapacidad } \\
\text { intelectual }\end{array}$ & $\begin{array}{l}\text { Es obligado tener presente la dimensión personal y subjetiva en el diseño y } \\
\text { realización de planes que están orientados a potenciar el desarrollo personal y las } \\
\text { competencias del individuo, siendo el bienestar emocional una dimensión clave e } \\
\text { irremplazable de la calidad de vida. }\end{array}$ \\
\hline Acosta (2011) & $\begin{array}{l}\text { Alumnos y alumnas } \\
\text { con discapacidad } \\
\text { intelectual }\end{array}$ & $\begin{array}{l}\text { El desarrollo de la educación emocional constituye un recurso para lograr una } \\
\text { educación de calidad basada en el desarrollo integral. Además, las personas con } \\
\text { coeficiente intelectual bajo, pero con conciencia emocional alta, pueden lograr una } \\
\text { vida "más feliz". }\end{array}$ \\
\hline Simón (2012) & $\begin{array}{l}\text { Estudiantes entre } \\
6 \text { y } 18 \text { años de } \\
\text { un centro de } \\
\text { Educación Especial, } \\
\text { con niveles } \\
\text { diferentes niveles } \\
\text { cognitivos (desde } \\
\text { severos hasta } \\
\text { límites) }\end{array}$ & $\begin{array}{l}\text { En las programaciones, con el objetivo de conseguir el máximo desarrollo de } \\
\text { las personas, deben desarrollarse las competencias básicas, entre ellas, la } \\
\text { competencia emocional. La educación de la misma, así como la enseñanza de } \\
\text { las habilidades sociales, hace mejorar su capacidad adaptativa (facilitando su } \\
\text { aceptación y convivencia en la sociedad), además de cumplir la función preventiva } \\
\text { ante situaciones de ansiedad, impulsividad, etc. Del mismo modo, el desarrollo } \\
\text { de la competencia emocional y, por lo tanto, la inteligencia emocional favorece la } \\
\text { convivencia en las instituciones educativas; motivo por el que deberían considerarse } \\
\text { programas de esta índole en los entornos educativos. }\end{array}$ \\
\hline
\end{tabular}




\begin{tabular}{|c|c|c|}
\hline AUTOR & MUESTRA & CONCLUSIONES \\
\hline Alonso (2013) & $\begin{array}{l}\text { Alumna de } 18 \text { años } \\
\text { con discapacidad } \\
\text { intelectual }\end{array}$ & $\begin{array}{l}\text { Se concluye que la intervención propuesta posibilita al alumnado advertir la utilidad } \\
\text { e importancia de la competencia emocional, fortaleciendo su participación en } \\
\text { la sociedad y favoreciendo su propio conocimiento; mejoran así, su desarrollo } \\
\text { y calidad de vida para desenvolverse en diversos contextos y facilitando su } \\
\text { integración e inclusión social. }\end{array}$ \\
\hline Cal (2014) & $\begin{array}{l}8 \text { personas con } \\
\text { discapacidad } \\
\text { intelectual de una } \\
\text { vivienda tutelada de } \\
\text { ámbito rural }\end{array}$ & $\begin{array}{l}\text { Hoy en día existe una gran necesidad de trabajar la inteligencia personal y la } \\
\text { gratitud de estas personas ya que, muchas de ellas, carecen o disponen de } \\
\text { una gama muy escasa de habilidades sociales, presentando una competencia } \\
\text { social y personal muy pobre. Así mismo, existe una gran necesidad de trabajar } \\
\text { el conocimiento emocional, tanto propio como ajeno, debido a los conflictos que } \\
\text { surgen por esta consecuencia. }\end{array}$ \\
\hline $\begin{array}{l}\text { Martín et al. } \\
\text { (2015) }\end{array}$ & $\begin{array}{l}\text { Personas con } \\
\text { discapacidad } \\
\text { intelectual }\end{array}$ & $\begin{array}{l}\text { El aspecto tanto emocional como social es conveniente para mejorar el ámbito } \\
\text { relacional de la persona, así como para acrecentar su confianza y seguridad } \\
\text { en sí mismo o misma. En este estudio se observa un aumento significativo de } \\
\text { los aspectos emocionales y sociales tras la puesta en marcha de un programa } \\
\text { especializado. }\end{array}$ \\
\hline $\begin{array}{l}\text { Robles-Bello } \\
\text { et al. (2015) }\end{array}$ & $\begin{array}{l}\text { Niños, niñas } \\
\text { y jóvenes con } \\
\text { síndrome de Down } \\
\text { con retraso leve } \\
\text { (10-23 años) }\end{array}$ & $\begin{array}{l}\text { Los jóvenes con síndrome de Down obtienen menores puntuaciones, por lo que } \\
\text { se considera la necesidad de incorporar entrenamiento en inteligencia emocional. } \\
\text { Además, en personas con desarrollo típico se ha encontrado un mayor nivel de } \\
\text { inteligencia emocional, relacionada con menores puntuaciones en ansiedad. Esto } \\
\text { último se desconoce en casos de personas con síndrome de Down pero diversos } \\
\text { estudios han descubierto que estas personas, con frecuencia, presentan altos } \\
\text { niveles de ansiedad. }\end{array}$ \\
\hline $\begin{array}{l}\text { Sheydaei et al. } \\
\text { (2015) }\end{array}$ & $\begin{array}{l}32 \text { estudiantes } \\
\text { con discapacidad } \\
\text { intelectual entre } 14 \\
\text { y } 18 \text { años }\end{array}$ & $\begin{array}{l}\text { Se descubrió que el entrenamiento de la inteligencia emocional procura habilidades } \\
\text { comunicativas a estos y estas estudiantes. Se demostró una relación entre ésta y } \\
\text { las habilidades comunicativas, lo que significa que, si una persona tiene la habilidad } \\
\text { de entender los cambios de ánimo de otros y otras, puede identificar, controlar y } \\
\text { manejar sus propias emociones y las de los demás. Además, se vio que existe una } \\
\text { relación también entre la mayoría de los componentes de la inteligencia emocional } \\
\text { con los problemas interpersonales, prediciendo que pueden sufrirse dificultades } \\
\text { interpersonales. Por ello, se demuestra que esta inteligencia reduce estos } \\
\text { problemas y mejora la cualidad de las relaciones sociales e interpersonales. }\end{array}$ \\
\hline $\begin{array}{l}\text { Barradas } \\
(2016)\end{array}$ & $\begin{array}{l}\text { Niños y niñas de } 6 \text { a } \\
11 \text { años. }\end{array}$ & $\begin{array}{l}\text { La competencia emocional de estos alumnos y alumnas aumentaba } \\
\text { significativamente después de la realización del programa. Además, por otra parte, } \\
\text { se observó que necesitan una intervención educativa en inteligencia emocional; por } \\
\text { el motivo de que, durante su educación, se habían dejado de lado los problemas } \\
\text { sobre las relaciones con sus pares o expresar fácilmente sus sentimientos. Se les } \\
\text { han proporcionado herramientas para cubrir sus necesidades sociales, reforzando } \\
\text { su autoeficacia y autoestima. También ha sido visible una mejora del clima de } \\
\text { trabajo en el aula. }\end{array}$ \\
\hline $\begin{array}{l}\text { Bravo et al. } \\
(2016)\end{array}$ & $\begin{array}{l}3 \text { jóvenes con } \\
\text { discapacidad } \\
\text { intelectual } \\
\text { moderada y una } \\
\text { con trastorno del } \\
\text { espectro autista } \\
\text { (14-18 años) }\end{array}$ & $\begin{array}{l}\text { Existe una mejora en la capacidad de autorregulación emocional, expresión } \\
\text { emocional y desarrollo de habilidades pragmáticas de estas jóvenes con } \\
\text { discapacidad intelectual, no surtiendo efecto alguno en la otra chica. La efectividad } \\
\text { del programa demuestra que puede ser de gran utilidad para el desarrollo de } \\
\text { competencias sociales y emocionales. }\end{array}$ \\
\hline $\begin{array}{l}\text { Cabezas } \\
(2016)\end{array}$ & $\begin{array}{l}\text { Personas con } \\
\text { discapacidad } \\
\text { intelectual }\end{array}$ & $\begin{array}{l}\text { Existen investigaciones y evidencias del efecto positivo de los programas de } \\
\text { educación emocional desarrollados en estas personas; procurando que gestionen } \\
\text { mejor su mundo emocional y, por consiguiente, ser más competentes para la vida y } \\
\text { gozar de mayor bienestar, contribuyendo especial y eficientemente en su desarrollo } \\
\text { personal. }\end{array}$ \\
\hline
\end{tabular}




\begin{tabular}{|c|c|c|}
\hline AUTOR & MUESTRA & CONCLUSIONES \\
\hline $\begin{array}{l}\text { Cabrera et al. } \\
\text { (2016) }\end{array}$ & $\begin{array}{l}\text { Niños y niñas } \\
\text { en condición de } \\
\text { discapacidad } \\
\text { intelectual }\end{array}$ & $\begin{array}{l}\text { Para que estos niños y niñas alcancen un desarrollo social que les permita } \\
\text { relacionarse es importante que en la familia y escuela se integren actividades que } \\
\text { les enseñen a comunicarse, a respetar y valorar las reglas conjuntas y a informar } \\
\text { sobre sus sentimientos de manera asertiva. }\end{array}$ \\
\hline $\begin{array}{l}\text { Collantes } \\
\text { (2016) }\end{array}$ & $\begin{array}{l}\text { Personas con } \\
\text { síndrome de Down }\end{array}$ & $\begin{array}{l}\text { A día de hoy, ayudar a los educandos (a partir de los } 2 \text { años) a desarrollar sus } \\
\text { habilidades emocionales y sociales propicia que crezcan sanos y que se sientan } \\
\text { mejor consigo mismos y mismas; ya que un niño o niña que conozca y sepa } \\
\text { gestionar sus emociones obtiene mejores resultados académicos y estará mejor } \\
\text { preparado para la vida. Además, puede beneficiarse de aspectos como: desarrollo } \\
\text { de pensamientos positivos, asunción de responsabilidad, comprensión de los y las } \\
\text { demás, etc. }\end{array}$ \\
\hline $\begin{array}{l}\text { Orellana- } \\
\text { Cortijo (2017) }\end{array}$ & $\begin{array}{l}18 \text { estudiantes } \\
\text { jóvenes } \\
\text { adolescentes, } \\
\text { entre } 16 \text { y } 21 \text { años, } \\
\text { con discapacidad } \\
\text { intelectual leve } \\
\text { de un centro de } \\
\text { Educación Especial }\end{array}$ & $\begin{array}{l}\text { El desarrollo de las aptitudes emocionales es imprescindible para llevar una vida } \\
\text { exitosa y plena. Por lo tanto, se hace necesario trabajar la inteligencia emocional } \\
\text { en la educación; ya que la falta de habilidades emocionales puede ocasionar } \\
\text { o facilitar la aparición de conflictos y problemas de conducta. Además, las } \\
\text { competencias afectivas y emocionales desempeñan un papel fundamental en } \\
\text { estos y estas estudiantes para adaptar sus emociones, relacionarse y aprender a } \\
\text { quererse y sentirse bien con ellos y ellas mismas. Es más, el desarrollo de la misma } \\
\text { deja evidencias de que mejora la conducta en el aula, con mayor tolerancia a la } \\
\text { frustración, mayor autocontrol, mejora en las habilidades asertivas, de relación } \\
\text { interpersonal y mayor participación, demostrando los efectos positivos provenientes } \\
\text { del entrenamiento en competencias emocionales. }\end{array}$ \\
\hline $\begin{array}{l}\text { Álvarez et al. } \\
\text { (2018) }\end{array}$ & $\begin{array}{l}\text { Niños y niñas } \\
\text { con necesidades } \\
\text { educativas } \\
\text { especiales }\end{array}$ & $\begin{array}{l}\text { No desarrollar la inteligencia emocional trae consigo aspectos como: frustración, } \\
\text { conductas desadaptativas e incluso bajo desempeño académico y crecimiento } \\
\text { personal. El desarrollo de la misma proporciona beneficios en diversas áreas } \\
\text { de desarrollo de los niños y niñas con necesidades especiales, permitiéndoles } \\
\text { que asimilen mejor sus emociones y las de los demás, generando crecimiento } \\
\text { emocional. Además de esto, dicha inteligencia es de vital importancia para mejorar } \\
\text { el clima escolar, además de procurar una optimización en las habilidades de los } \\
\text { niños y niñas, que les permite desenvolverse mejor. }\end{array}$ \\
\hline $\begin{array}{l}\text { Cabezas et al. } \\
\text { (2018) }\end{array}$ & $\begin{array}{l}\text { Estudiantes con } \\
\text { discapacidad } \\
\text { intelectual leve y } \\
\text { moderada }\end{array}$ & $\begin{array}{l}\text { Potenciar la inteligencia emocional contribuye a obtener individuos emocionalmente } \\
\text { autónomos, capaces de tomar sus decisiones. Ayuda también a superar episodios } \\
\text { de ansiedad y/o estrés, así como a controlar impulsos y favorece el bienestar } \\
\text { psicológico y personal, ya que se incentiva su desarrollo personal y social, } \\
\text { incrementando sus habilidades prácticas y sociales. }\end{array}$ \\
\hline Méndez (2018) & $\begin{array}{l}3 \text { alumnos entre } 3 \text { y } \\
12 \text { años }\end{array}$ & $\begin{array}{l}\text { La inteligencia emocional participa en el proceso de desarrollo de cada persona. } \\
\text { Ayuda a identificar emociones mediante el desarrollo de habilidades sociales. Las } \\
\text { dificultades encontradas de estos tres estudiantes se encuentran en la fase de } \\
\text { identificación de las emociones de los demás, por lo que se ve necesario realizar } \\
\text { más programas relacionados con el desarrollo de la inteligencia emocional. }\end{array}$ \\
\hline Puzdre (2018) & $\begin{array}{l}\text { Personas con } \\
\text { discapacidad } \\
\text { intelectual }\end{array}$ & $\begin{array}{l}\text { Se demuestra que la competencia emocional influye enormemente en la adquisición } \\
\text { de los contenidos curriculares. Y esto es válido para todas las personas, no } \\
\text { únicamente para las que tienen discapacidad intelectual. }\end{array}$ \\
\hline $\begin{array}{l}\text { Sánchez- } \\
\text { Ramírez (2018) }\end{array}$ & $\begin{array}{l}18 \text { personas } \\
\text { usuarias de un } \\
\text { centro de día con } \\
\text { discapacidad } \\
\text { intelectual } \\
\text { moderada o ligera } \\
\end{array}$ & $\begin{array}{l}\text { Se han obtenido resultados que muestran la existencia de diferencias positivas } \\
\text { antes y después del tratamiento. El programa ha mejorado los niveles de percepción } \\
\text { y comprensión de sus participantes; corroborando los resultados positivos de } \\
\text { otros problemas sobre la inteligencia emocional y este colectivo (esta capacidad se } \\
\text { puede aprender y mejorar). Es más, ésta influye en la vida social, satisfacción vital y } \\
\text { resolución de problemas, y, de manera general, en el bienestar. }\end{array}$ \\
\hline $\begin{array}{l}\text { Soler et al. } \\
(2018)\end{array}$ & $\begin{array}{l}\text { Personas adultas } \\
\text { con discapacidad } \\
\text { intelectual }\end{array}$ & $\begin{array}{l}\text { Existen estudios que evidencian la relación entre la autoestima y la inteligencia } \\
\text { emocional, mostrando una correlación positiva entre esta última y el bienestar } \\
\text { personal, así como relaciones significativas con el autocontrol emocional. }\end{array}$ \\
\hline
\end{tabular}




\begin{tabular}{|l|l|l|}
\hline AUTOR & MUESTRA & CONCLUSIONES \\
\hline Suriá (2018) & $\begin{array}{l}\text { Universitarios } \\
\text { y universitarias } \\
\text { entre } 18 \text { y } 33 \text { años } \\
\text { con diferentes } \\
\text { tipos y grados de } \\
\text { discapacidad }\end{array}$ & $\begin{array}{l}\text { Muchos autores indican que los universitarios y universitarias padecen mayores } \\
\text { dificultades, ya que no solo tienen que superar barreras arquitectónicas y/o } \\
\text { curriculares sino que también existen barreras actitudinales hacia ellos y ellas por } \\
\text { parte de los y las demás, así como los propios obstáculos internos que se pone } \\
\text { cada sujeto. El perfil de inteligencia emocional de una persona con discapacidad } \\
\text { intelectual está caracterizado por baja atención emocional, aunque alta claridad y } \\
\text { reparación emocional, aspecto que hace comprobar la necesidad de desarrollo que } \\
\text { tienen en una de las áreas de la inteligencia emocional. }\end{array}$ \\
\hline $\begin{array}{l}\text { Caro et al. } \\
\text { (2019) }\end{array}$ & $\begin{array}{l}\text { Personas con } \\
\text { discapacidad } \\
\text { intelectual }\end{array}$ & $\begin{array}{l}\text { La educación emocional es un proceso continuo y permanente que potencia el } \\
\text { desarrollo de las competencias emocionales como elemento del desarrollo integral } \\
\text { de una persona, para capacitarla de cara a afrontar mejor los retos que se le } \\
\text { presenten en la vida cotidiana. }\end{array}$ \\
\hline $\begin{array}{l}\text { Gavín- } \\
\text { Chocano y } \\
\text { Molero (2019) }\end{array}$ & $\begin{array}{l}\text { Personas con } \\
\text { discapacidad } \\
\text { intelectual leve (15 } \\
\text { sujetos con una } \\
\text { media de edad de } \\
\text { 34,93 años) }\end{array}$ & $\begin{array}{l}\text { Investigaciones demuestran que estas personas son capaces de identificar } \\
\text { y describir estrategias para afrontar circunstancias estresantes, teniendo la } \\
\text { capacidad de superarlas. Sin embargo, han de intensificarse la adquisición de } \\
\text { sus competencias emocionales; debido a que, de este modo, se incrementaría la } \\
\text { posibilidad de afrontar situaciones adversas proporcionando mayor satisfacción } \\
\text { vital. Así mismo, las habilidades emocionales desarrolladas les proporcionarían un } \\
\text { mayor nivel de autonomía y autogestión. }\end{array}$ \\
\hline
\end{tabular}

Fuente: elaboración propia.

\section{Discusión y conclusiones}

A través de la investigación realizada se ha comprobado la necesidad de introducir en las aulas programas relacionados con la educación emocional y el desarrollo de la inteligencia emocional, pudiéndose obtener ciertas mejoras sustanciales en los aspectos referidos a la percepción y expresión emocional, así como a su facilitación emocional, comprensión emocional y regulación emocional; a pesar de que, depende en mayor o menor medida en el grado o tipo de discapacidad intelectual que presente la persona en cuestión.

La necesidad de incorporar la inteligencia emocional y su desarrollo en las aulas para alumnado con necesidades especiales de tipo intelectual se justifica por los beneficios que puede ofrecer: proporcionarles una adecuada consolidación en su formación y desarrollo integral, calidad de vida y bienestar personal, crecimiento personal y conocimiento de sus propias emociones y ajenas, buscando ser más competentes para vivir en la sociedad actual. Una adecuada educación emocional, la cual intensificará y desarrollará la inteligencia emocional de este colectivo, les procuraría la oportunidad de conocer mejor sus emociones, identificándolas, diferenciándolas y asimilándolas; siendo capaces de expresar, controlar, adaptar y gestionar sus propias emociones y las ajenas. Del mismo modo, estas personas podrían gozar de un bienestar tanto emocional como personal, que, a su vez, les permitiría enfrentarse a diferentes hándicaps y necesidades de manera más efectiva, como la confianza y seguridad que les proporciona este tipo de inteligencia para afrontar su posible ansiedad, frustración y conductas desadaptativas, así como la autoeficacia y autoestima que les puede llegar a dar. Por tanto, la inteligencia emocional también les ayudaría a afrontar de manera 
más autónoma y eficaz los retos que se propongan, permitiéndoles, desenvolverse mejor y que tomen sus propias decisiones, sobreponiéndose a los problemas y dificultades con mayor responsabilidad, optimismo y pensamientos más positivos.

Por último, entre los resultados obtenidos cabría destacar la visible mejora en uno de los hándicaps que presentan, como son las habilidades adaptativas, junto con el desarrollo de habilidades pragmáticas. Así mismo, sus competencias sociales y emocionales se acrecientan y desarrollan procurando relacionarse mejor y potenciando sus habilidades asertivas. No obstante, pueden solventarse los frecuentes problemas de índole interpersonal que suelen sufrir estas personas, dando fruto a una convivencia y vida social más sana, con mejores habilidades comunicativas, y de comprensión, pudiendo tener la oportunidad de desenvolverse socialmente. En el ámbito académico y escolar, también se han podido observar efectos positivos en los resultados, como la mejora del clima del aula, fortaleciendo la participación de estos estudiantes, la prevención de posibles conflictos, disputas, discriminación, la falta de entendimiento, etc., buscando alcanzar la concienciación sobre las propias emociones y el control de las mismas (Jiménez y López-Zafra, 2009), y también el bienestar subjetivo con estrategias de regulación emocional (Extremera et al., 2020). Sin embargo, todo lo mencionado hasta ahora puede verse influenciado por el tipo y grado de discapacidad intelectual de cada sujeto, aunque es innegable la cuantía de aspectos positivos de los que podría beneficiarse cada persona en mayor o menor medida. No obstante, se corrobora la necesidad e importancia de introducir una educación emocional y el consiguiente desarrollo de la inteligencia emocional para estas personas por los ámbitos que podría mejorar o beneficiarse.

Por tanto, las instituciones educativas deberían incluir y potenciar la inteligencia emocional como parte de la enseñanza de forma permanente (Álvarez et al., 2018; Fiori y Vesely-Maillefer, 2018), convirtiéndose ésta en imprescindible para el desarrollo integral de cualquier persona (Collantes, 2016). Las emociones constituyen un factor muy importante para estas personas pues potenciando su inteligencia emocional podrían alcanzar un equilibrio emocional que favorecería su adaptación al entorno (Cal, 2014). Así mismo, según Ruiz (2004) las emociones son estados de un individuo, y los sujetos con necesidades especiales de tipo intelectual tienen una vida emocional tan rica como las de los demás; viviendo los efectos de esos sentimientos igual o incluso con mayor intensidad que las personas que les rodean. Estas personas pueden tener ciertas dificultades a la hora de controlar sus emociones (Álvarez et al., 2018), así como las limitaciones presentes en las habilidades adaptativas (conformadas por áreas como la comunicación, el autocuidado, la seguridad...), que repercuten en sus habilidades y relaciones sociales, haciendo que se conviertan en un grupo vulnerable y de riesgo (Simón, 2012). Todo esto impide que la persona pueda funcionar adecuadamente en su vida diaria, incapacitando su funcionamiento acorde con lo esperado, impidiendo responder apropiadamente a cambios vitales o a las demandas del ambiente, etc. (Cabezas et al., 2018). Estas dificultades, que les llevan a una inmadurez social por la falta de comprensión de valores, normas y relaciones, pueden desembocar en conflictos y, en ocasiones, episodios de agresividad a la hora de intentar resolver problemas (Rodríguez, 2008).

Algunos estudiantes diagnosticados de discapacidad intelectual presentan dificultades para relacionarse con sus pares debido a cómo manejan sus emociones (Cabrera et al., 2016). Por tanto, considerando todo lo mencionado hasta ahora, la educación emocional y el desarrollo y fomento de la inteligencia emocional permiten asegurar un mejor conocimiento de las propias emociones, así como un dominio adecuado de las mismas para que una persona pueda llevar a cabo su proyecto de vida. Es más, estas personas con necesidades especiales, en ocasiones, pueden tener mayores dificultades para inhibir o regular sus emociones. Este es un aspecto que ha de trabajarse, para un adecuado desenvolvimiento social y autocontrol (Ruiz, 
2004); viéndose esencial trabajar las habilidades emocionales o la competencia emocional para fortalecer y promulgar dicha educación entre estos estudiantes, procurando del mismo modo una educación y desarrollo integral (Alonso, 2013) a través de la inteligencia emocional. 
Referencias bibliográficas

Acosta, M. N. (2011). Inteligencia emocional y déficit mental. Innovación y experiencias educativas, 38, pp. 1-8.

Alonso, J. (2013). Competencia emocional en alumnado con discapacidad intelectual: propuesta de intervención educativa [Trabajo de Fin de Grado]. Universidad de Valladolid.

Álvarez, K. A. et al. (2018). Inteligencia emocional en niños con necesidades educativas especiales [Trabajo de Fin de Grado]. Universidad Estatal de Milagro.

Aránega, I. M. et al. (2014). Reconocimiento de emociones en el alumnado de Educación Infantil en situaciones cognitivas y en figuras faciales. En P. Miralles et al. (Eds.), Investigación e innovación en Educación Infantil (pp. 107-116). Universidad de Murcia.

Bar-On, R. (1997). The Emotional Quotient Inventory (EQ-i): A test of emotional intelligence. Multi-Health Systems.

Barradas, T. (2016). Propuesta de programa de intervención en inteligencia emocional adaptado a niños y niñas entre 12 y 15 años con necesidades especiales [Trabajo de Fin de Grado]. Universidad Miguel Hernández.

Bisquerra, R. et al. (2015). Inteligencia emocional en educación. Editorial Síntesis.

Buitrago, R. E. y Herrera, L. (2013). Matricular las emociones en la escuela, una necesidad educativa y social. Praxis \& Saber, 8, pp. 87-108.

Braslavsky, C. (2004). Diez factores para una educación de calidad para todos en el siglo XXI. Santillana.

Bravo, M. A. et al. (2016). Desarrollo de habilidades comunicativas pragmáticas y autorregulación emocional en alumnos con discapacidad intelectual y trastorno del espectro del autismo. Ediciones Universidad de San Jorge.

Cabezas, D. (2016). Inteligencia intrapersonal y desarrollo en las personas con síndrome de Down. Revista Síndrome de Down, 33, pp. 116-130.

Cabezas, N. et al. (2018). Incidencia de los factores afectivos en el aprendizaje de estudiantes con Discapacidad Intelectual, pertenecientes a establecimientos en situación de vulnerabilidad de la ciudad de Los Ángeles durante el año 2018 [Tesis de Pregrado]. Universidad de Concepción.

Cabrera, V. E. et al. (2016). Necesidades de relaciones sociales de niños y niñas con discapacidad intelectual en la familia y en la escuela. Educación y Desarrollo Social, 10(2), pp. 86-101.

Cal, A. (2014). Psicología positiva en la discapacidad intelectual. Programa para el desarrollo emocional y la fortaleza de gratitud [Trabajo de Fin de Master]. Universidad de Valladolid.

Caro, M. A. et al. (2019). Inteligencia emocional y educación emocional en personas en situación de discapacidad intelectual [Tesis doctoral]. Universidad Gabriela Mistral.

Chamarro, A. y Oberst, U. (2004). Modelos teóricos en inteligencia emocional y su medida. Aloma, 14, pp. 209217.

Collantes, P. (2016). Educación emocional y discapacidad [Trabajo de Fin de Grado]. Universidad de Valladolid.

Delors, J. (1996). La educación encierra un tesoro. Santillana.

España. Ley Orgánica 8/2013, de 9 de diciembre, para la mejora de la calidad educativa. Boletín Oficial del Estado, 10 de diciembre de 2013, núm. 295, pp. 97858-97921. 
España. Ley Orgánica 3/2020, de 29 de diciembre, por la que se modifica la Ley Orgánica 2/2006, de 3 de mayo, de Educación. Boletín Oficial del Estado, 30 de diciembre de 2020, núm. 340, pp. 122868-122953.

Extremera, N. et al. (2020). Pathways between ability emotional intelligence and subjective well-Being: Bridging links through cognitive emotion regulation strategies. Sustainability, 12(5), pp. 1-11. https://doi.org/10.3390/ su12052111.

Fernández-Berrocal, P. y Extremera, N. (2002). La inteligencia emocional como una habilidad esencial en la escuela. Revista Iberoamericana de Educación, 29(1), pp. 1-6.

Fernández-Berrocal, P. y Extremera, N. (2005). La inteligencia emocional y la educación de las emociones desde el Modelo de Mayer y Salovey. Revista Interuniversitaria de Formación del Profesorado, 19(3), pp. 63-93.

Fiori, M. y Vesely Maillefer A. (2018). Emotional intelligence as an ability: Theory, challenges, and new directions. En K. V. Keefer et al. (Eds.), Emotional intelligence in Education. Springer.

Fogarty, G. J. et al. (2016). Evaluating measures of optimism and sport confidence. Measurement in Physical Education and Exercise Science, 20(2), pp. 81-92. http://dx.doi.org/10.1080/1091367X.2015.1111220.

García-Tudela, P. A. y Marín-Sánchez, P. (2021). Educación de la inteligencia emocional en edad escolar: un estudio exploratorio desde la perspectiva docente. Revista Electrónica Educare, 25(3), pp. 1-21.

Garrote et al. (2019). El trabajo cooperativo como herramienta formativa en los estudiantes universitarios. Revista Iberoamericana sobre Calidad, Eficacia y Cambio en Educación, 17(3), pp. 41-58.

Gavín-Chocano, O. y Molero, D. (2020). Valor predictivo de la inteligencia emocional percibida y calidad de vida sobre la satisfacción vital en personas con discapacidad intelectual. Revista de Investigación Educativa, 38(1), pp. 131-148. https://doi.org/10.6018/rie.331991.

Gento, S. y Strnadová, I. (2011). Tratamiento educativo de la diversidad intelectual. UNED.

Goleman, D. (1995). Inteligencia emocional. Editorial Kairós.

González, E. (2009). Evolución de la Educación Especial: del modelo del déficit al modelo de la escuela inclusiva. El largo camino hacia una educación inclusiva: la educación especial y social del siglo XIX a nuestros días. XV Coloquio de Historia de la Educación, Pamplona-Iruña, 29, 30 de junio y 1 de julio de 2009, 1, pp.429-440.

González, R. y Villanueva, L. (2014). Recursos para educar en emociones. De la teoría a la acción. Pirámide.

Jiménez M. I. y López-Zafra, E. (2009). Inteligencia emocional y rendimiento escolar: estado actual de la cuestión. Revista latinoamericana de Psicología, 41(1), pp. 69-79.

Martín, L. et al. (2015). Desarrollo emocional y social en un grupo de personas con discapacidad intelectual. Cuadernos de Psiquiatría y Psicoterapia del Niño y del Adolescente, 60, pp. 45-51.

Mateo, F. (2015). Producción científica en español en humanidades y ciencias sociales: algunas propuestas desde Dialnet. El profesional de la información, 24(5), pp. 509-516.

Mateu, G. (1987). Educar para la felicidad. Colección Azenai.

Mayer, J. D. et al. (1999). Emotional intelligence meets traditional standards for an intelligence. Intelligence, 27, pp. 267-298.

Mayer, J. D. y Salovey, P. (1997). Emotional development and emotional intelligence: Educational implications. Basic Books. 
Mella, O. y Ortiz, I. (1999). Rendimiento escolar. Influencias diferenciales de factores externos e internos. Revista Latinoamericana de Estudios Educativos, 1, pp. 69-92.

Méndez, A. (2018). Propuesta de intervención sobre inteligencia emocional en alumnos con discapacidad [Trabajo de Fin de Grado]. Universidad de Valladolid.

Mestre-Navas, J. y Fernández-Berrocal, P. (2007). Manual de inteligencia emocional. Pirámide.

Orellana-Cortijo, M. B. (2017). Intervención para la mejora de la Inteligencia Emocional en adolescentes con Discapacidad intelectual [Tesis de Máster]. Universidad Internacional de la Rioja.

Palomera, R. et al. (2017). La inteligencia emocional como una competencia básica en la formación inicial de los docentes: algunas evidencias. Electronic Journal of Research in Education Psychology, 6(15), pp. 72-77.

Paredes, D. (2010). Bienestar emocional y expresión conductual en las personas con discapacidad intelectual. Educación y Diversidad, 4(2), pp. 51-60.

Pinos, M. (2019). Con corazón y cerebro. Net learning: aprendizaje basado en la neurociencia, la emoción y el pensamiento. Editorial Caligrama.

Puzdre, M. M. (2018). Trabajo de la inteligencia emocional en personas con discapacidad intelectual, a través de la música. Sentio, ergo sum [Trabajo de Fin de Grado]. Universidad de Valladolid.

Roberts, R. D. et al. (2001). Does emotional intelligence meet traditional standards for an intelligence? Some new data an conclusions. Emotion, 1, pp. 196-321.

Robles-Bello, M. A. et al. (2014). Inteligencia emocional en síndrome de Down. Avances en el estudio de la motivación y de la emoción. VIII Simposio de la Asociación de Motivación y Emoción (15, 16 y 17 de Mayo de 2014), pp. 46-50.

Rodríguez, M. S. (2008). Educar en las emociones: un desafío hacia la integración. UNIFE, 16(1), pp. 195-218.

Román, M. (2005). Sociedad del conocimiento y refundación de la escuela desde el aula. EOS.

Ruiz, A. B. (2010). Evolución de la educación. Pedagogía Magna, 5, pp. 27-35.

Ruiz, E. (2004). Programa de educación emocional para niños y jóvenes con síndrome de Down. Revista Síndrome de Down, 21(82), pp. 84-93.

Salovey, P. y Mayer, J. D. (1990). Emotional intelligence. Imagination, Cognition and Personality, 9, pp. $185-211$.

Sánchez-Ramírez, N. (2018). Efectos de un programa de educación en inteligencia emocional sobre la percepción y la comprensión emocional en personas con discapacidad intelectual [Trabajo de Fin de Máster]. Universidad Internacional de la Rioja.

Shalock, R. L. (2009). La nueva definición de discapacidad intelectual, apoyos individuales y resultados personales. Revista Española sobre Discapacidad Intelectual, 229(40), pp. 22-39.

Sheydaei, M. et al. (2015). The effectiveness of emotional intelligence training on communication skills in students with intellectual disabilities. Iranian Rehabilitation Journal, 16(3), pp. 7-12.

Simón, E. M. (2012). Educación emocional y habilidades sociales con alumnos con necesidades educativas especiales: propuesta de intervención [Trabajo de Fin de Grado]. Universidad de Valladolid.

SOLER, J. L. et al. (2018). Inteligencia emocional y bienestar III. Reflexiones, experiencias profesionales e investigación. Asociación Aragonesa de Psicopedagogía. 
Sternberg, R. J., y Kaufman, J. C. (1998). Human abilities. Annual review of psychology, 49(1), pp. 479-502.

Suriá, R. (2018). Inteligencia emocional y bienestar subjetivo en estudiantes universitarios con diferentes tipos y grados de discapacidad. Bordón. Revista de Pedagogía, 70(1), pp. 125-140.

Trujillo, M. y Rivas, L. (2005). Orígenes, evolución y modelos de inteligencia emocional. INNOVAR. Revista de Ciencias Administrativas y Sociales, 15(25), pp. 16-19.

Zubiri, X. (2006). Tres dimensiones del ser humano: individual, social, histórica. Editorial. 